\title{
Heart rate variability and long chain n-3 PUFA in patients with chronic kidney disease commencing haemodialysis: a cross-sectional study
}

\section{Abstract}

The risk of sudden cardiac death (SCD) is doubled when a patient with chronic kidney disease (CKD) stage 5 starts haemodialysis. Low heart rate variability (HRV) has been reported to be independently associated with increased risk of SCD and all cardiac death in haemodialysis patients. Long chain n-3 polyunsaturated fatty acids (LC n-3 PUFA; 20:5n-3, EPA and 22:6n-3, DHA) may exert antiarrhythmic effects on cardiac myocytes. Haemodialysis patients have lower serum LC n-3 PUFA levels compared to populations without CKD. Few studies have investigated the relationship between LC n-3 PUFA and HRV in patients with CKD. This study aimed to characterise the variability of LC n-3 PUFA status in patients who recently commenced haemodialysis, and to investigate relationships between LC n-3 PUFA status and HRV. A cross-sectional study was conducted in adults aged 40-80 years with CKD commencing haemodialysis (within 6-10 weeks) (NRES research ethics committee ref: 14/LO/0186). At 2 separate study days, predialysis blood samples were taken to measure fatty acid composition by GC, and HRV monitors (Actiheart, CamNtech Ltd, UK) were fitted after dialysis had started to monitor parameters of cardiac autonomic function during dialysis, during the night, and for a total of $24 \mathrm{~h}$. Forty-five patients (mean age 58 y, SD 9, 20 females $/ 25$ males) completed data collection at least once; $91 \%$ presented hypertension and 39\% had type 2 diabetes. Sample mean omega-3 index (O3I; EPA + DHA as a \% of fatty acids in erythrocyte membranes) was very low (3.45\%, SD 1.25 ; median $3.26 \%$, IQR 1.32$)$; only 2 individuals had O3I $>5 \%$. Variability in erythrocyte EPA (median $0.66 \%$, IQR 0.42) and DHA (median $2.40 \%$, IQR 1.32) was limited. Most HRV parameters did not significantly correlate with O3I following adjustment (e.g. age, BMI, $\beta$-blockers). Plasma EPA significantly positively correlated with overall and longer phase components of HRV and significantly negatively correlated with beat-to-beat variability over $24 \mathrm{~h}$ after full adjustment for confounders. This suggests that although higher circulating EPA concentrations were associated with better cardiac responsivity to environmental stimulations over $24 \mathrm{~h}$, they were also associated with poorer parasympathetic tone (the predominant influence on beat-to-beat HRV). No correlations were observed between plasma DHA and HRV. The divergent pattern of relationships between plasma EPA versus DHA and HRV raises the theory that patients commencing haemodialysis may have compromised conversion of EPA to DHA which may impair vagally-mediated regulation of cardiac autonomic function, a potential mechanism for high risk of SCD.

Conflict of Interest

There is no conflict of interest 